\title{
Une nouvelle source cohérente UVX : la génération d'harmoniques d'ordre élevé d'un rayonnement laser intense
}

\section{A. L'Huillier et P. Balcou}

Service des Photons, Atomes et Molecules, Centre d'Etudes de Saclay, 91191 Gif sur Yvette Cedex, France

Noble gas atoms irradiated by intense laser pulses, in the range $10^{13}$ to 1015 $\mathrm{W} / \mathrm{cm}^{2}$, generate harmonics of the laser frequency up to a very high order. Radiations of about $150 \mathrm{eV}$ have been demonstrated, and up to $400 \mathrm{eV}$ are expected. As these harmonics are generated in short pulses (from $100 \mathrm{fs}$ to $30 \mathrm{ps}$ ), large instantaneous powers may be obtained: $3 \mathrm{~kW}$ have been measured in Saclay. This XUV source preserves the coherence properties of the pump laser and could find numerous applications thanks to its focusability, brightness and spectral narrowness.

Lorsqu'un laser de grande puissance est focalisé dans un jet atomique (à une pression d'une dizaine de Torr), des harmoniques impairs de la fréquence fondamentale sont générés. Ces processus de conversion de fréquence, étudiés dès les années 70 , connaissent aujourd'hui un regain d'intérêt avec l'utilisation de lasers de courte durée d'impulsion. Lorsque l'éclairement laser est peu intense, l'intensité des harmoniques décroît rapidement avec l'ordre du processus et il est difficile d'aller au delà des harmoniques 5 ou 7 . Lorsque l'éclairement laser est suffisamment intense pour induire une réponse non-perturbative de l'atome au champ de radiation, l'intensité des harmoniques devient constante, jusqu' à un ordre extrêmement élevé [1-4]. L'ordre maximum observé est de 109 à l'université de Stanford [5], à partir d'un laser Titane-Saphir à $800 \mathrm{~nm}$. L'énergie du rayonnement émis atteint $170 \mathrm{eV}(7.3 \mathrm{~nm})$. Un groupe de l'Université de Tokyo a observé l' harmonique 25 d'un laser excimere de longueur d'onde $248 \mathrm{~nm}[6]$, soit une Énergie de $125 \mathrm{eV}$. A Saclay, nous en sommes à l'harmonique 53 d'un laser à Néodyme de 1 ps [4], soit $62 \mathrm{eV}(20 \mathrm{~nm})$. La figure ci-contre montre les nombres de photons obtenus dans le néon et le xénon:

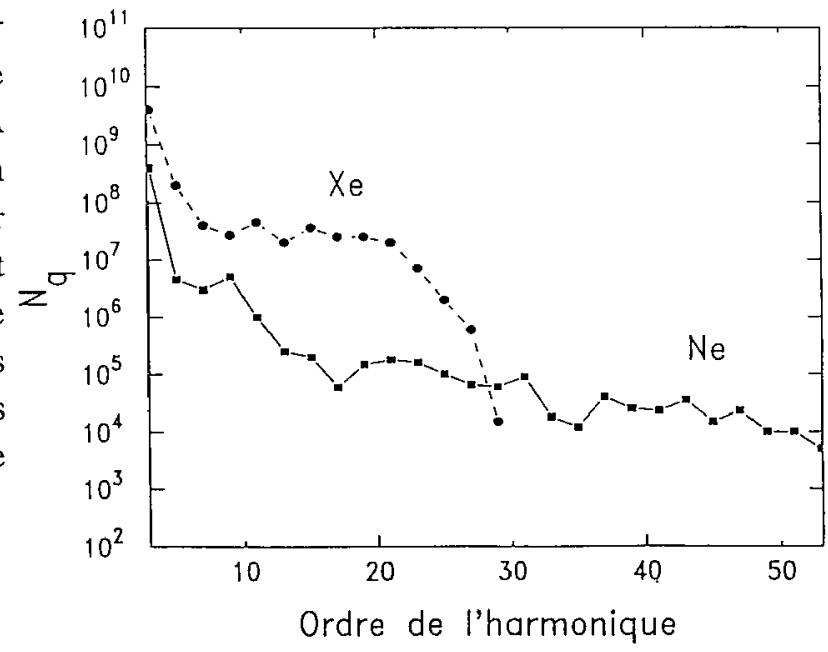


Le rayonnement harmonique possède les mêmes propriétés de cohérence et de divergence que celles d'un laser. La durée de l'impulsion suit (en étant légèrement inférieure) celle du laser incident. Si l'on utilise des lasers de très courte durée d'impulsion comme les lasers Titane-Saphir, on obtient des impulsions UVX de durée très brève, inférieure à $100 \mathrm{fs}$. Le rayonnement peut être accordable si le laser de pompe l'est. Une des caractéristiques les plus intéressantes du rayonnement harmonique est sa puissance instantanée et par conséquent sa brillance. Nous avons pu mesurer une puissance d'environ 3000 Watts à $20 \mathrm{eV}$, avec un éclairement laser incident de seulement $2 \times 10^{14} \mathrm{~W} / \mathrm{cm}^{2}$. Le rendement de conversion est de $5 \times 10^{-7}$. Partant d'un laser d'une puissance d'1 Térawatt, on peut espérer produire un rayonnement harmonique d'une puissance de l'ordre d' 1 Mégawatt. Enfin, ce rayonnement possédant les mêmes propriétés de cohérence spatiale que celles d'un laser, va pouvoir être focalisé sur une tache très petite, proche de la limite de diffraction, c'est-à-dire bien inférieure au micron. Par exemple, le diamètre de cette tache sera d'une centaine de nanomètres pour un rayonnement de $100 \mathrm{eV}$ focalisé à f/10. Dans la table, nous résumons les caracteristiques de l'émission à la 19 ième harmonique d'un laser à verre dopé au Néodyme généré dans un jet de xénon, en utilisant des données soit mesurées experimentalement (pour le nombre de photons) soit calculées (pour les largeurs temporelles et les dimensions, et donc pour la brillance instantanée) [3]. Nous pensons que ces valeurs pourront être étendues à des harmoniques plus élevés (et donc à un rayonnement de plus courte longueur d'onde) en utilisant des gaz plus légers comme le néon ou l'hélium et en optimisant la géométrie de l'interaction. La génération d'harmoniques élevés pourra ainsi être prochainement utilisée comme source cohérente UVX.

Table: 19ième harmonique généré dans le xénon d'un laser Néodyme à dérive de fréquence $(1,053 \mu \mathrm{m})$.

\begin{tabular}{|c|c|c|c|}
\hline Energie de photons & $22 \mathrm{eV}$ & Durée de l'impulsion & $0,5 \mathrm{ps}$ \\
\hline Largeur spectrale & $1 \AA$ & $\Delta \mathrm{E} / \mathrm{E}$ & $2 \times 10^{-3}$ \\
\hline Divergence & $1 \mathrm{mrad}$ & Photons / impulsion & $4 \times 10^{8}$ \\
\hline Photons/s.Å.(mrad)2 & 1021 & Dimension de la source & $1 \mu \mathrm{m}$ \\
\hline Puissance instantanée & $3 \mathrm{~kW}$ & Energie & $1,5 \mathrm{~nJ}$ \\
\hline Cadence & $1 \mathrm{tir} / 10 \mathrm{~s}$ & Eclairement & $4 \times 10^{11} \mathrm{~W} / \mathrm{cm}^{2}$ \\
\hline
\end{tabular}

\section{Références :}

[1] A. McPherson et al., J. Opt. Soc. Am. B 4, 595 (1987).

[2] X. F. Li, A. L'Huillier, M. Ferray, L. A. Lompré, G.Mainfray and C. Manus, Phys. Rev. A 39, 5751 (1989).

[3] A. L'Huillier et al., J. Phys. B: At. Mol. Opt. Phys. 24 (1991) 3315-3341.

[4] A. L'Huillier, L. A. Lompré, G. Mainfray and C. Manus, in "Atoms in

Intense Laser Fields", Ed. M. Gavrila, Academic Press, Orlando, Florida (à paraître dans la série "Advances in Optical, Atomic and Molecular Physics").

[5] J. J. Macklin, J. D. Kmetec and C. L. Gordon III, à paraître.

[6] N. Sarakura, K. Hata, T. Adachi, R. Nodomi, M. Watanabe and S. Watanabe, Phys. Rev. A 43, 1669 (1991). 